\title{
Optoelectronic Effect of Reflection Wall on LED Packaging Process
}

\author{
Wen-Ray Chen ${ }^{1 *}$ and De-Min $\mathrm{Wu}^{2}$ \\ ${ }^{1}$ Department of Electronic Engineering, National Formosa University, Yunlin 632, Taiwan \\ ${ }^{2}$ Department of Electro-Optical Engineering \& Graduate Institute of Electro-Optical and Materials Science, \\ National Formosa University, Yunlin 632, Taiwan
}

(Received October 31, 2017; accepted March 7, 2018)

Keywords: flashlight, SMD LED package

Currently, surface mount device (SMD) LED packages are widely used in many products, such as lighting, display backlights, cell phones, and automobiles. Therefore, in the supply terminal, the optimization of the packaging process is inevitable. It is important to enhance the ratio of performance to price and to match the customers' requirements for specific applications. Here, three types of LED packages were proposed. Their luminous flux, luminous efficacy, and illuminance were measured and discussed. The lumen value for the white wall sample increases by about $10.5 \%$ compared with that without the white wall. In addition, the enhanced white wall sample significantly reduces the usage of phosphors by about $25 \%$.

\section{Introduction}

In recent years, high-power white light-emitting diodes (WLED) have found many applications with surface mount technology. In particular, they were employed in flashlights of cell phones because of the demand for high light intensity in the normal direction. However, a high junction temperature of the LED was found when we turn the device into a super bright flashlight. Thus, substrates with high thermal conductivity were, in general, adopted as the thermal spreading material. For example, the low-temperature cofired ceramic (LTCC) substrate, ${ }^{(1,2)}$ AlN ceramic substrate, and diamond-like carbon (DLC) substrate were the most popular for the high-power LED application. Furthermore, to achieve high current spreading, the flip-chip technique ${ }^{(3,4)}$ was usually performed to reduce the series resistance and thermal resistance owing to the sapphire substrate used underneath the LED. In general, the driving current used in the flashlight was larger than that used in the lighting system. For example, a 40 mil square blue LED die was operated at a current of $350 \mathrm{~mA}$ that was generally used in the lighting module. However, it was operated at a current of $1 \mathrm{~A}$ while used in the flashlight. Fortunately, the turn-on time is very short (less than one second) when it was used in the flashlight. Thus, when the LED was operated at almost three times the standard operation condition, it might have some other applications such as in flashlight communication ${ }^{(5)}$ or

*Corresponding author: e-mail: chenwr@nfu.edu.tw

http://dx.doi.org/10.18494/SAM.2018.1794 
industrial lighting modules ${ }^{(6)}$ where an extra active cooling system needs to be used.

For the flashlight application, another important issue is the leakage of blue light underneath the phosphor paste. This is an issue caused by the packaging process. The light shape will thus appear as a blue ring, which results in a low color rendering index.

In this paper, to overcome the above problem, we focused on the flashlight of the cell phone. Here, three types of LED packages were proposed, and the optical parameters were determined and discussed in detail.

\section{Experimental Procedure}

In the experimental packaging process, the LED dies were eutectically bonded on top of the ceramic substrate. Then, a Zener diode was also bonded near the LED die using Ag paste. The curing process was performed at $150{ }^{\circ} \mathrm{C}$ for $2 \mathrm{~h}$. After the wire bonding was carried out for the Zener diode, the next step was the molding process.

Because the ceramic-based lead frame has a dimension of $2.0 \times 1.6 \mathrm{~mm}^{2}$, here, the LED flash product was denoted as 2016. The phosphor paste was sprayed onto the sample and the thickness was controlled at about $300 \mu \mathrm{m}$. In order to achieve a high color rendering index $(R a)$, the ratio of the blue/red phosphor was set as 80 throughout the experiments. Using the above controlling parameter, the average color rendering index can be obtained as high as $90 \%$.

The 2016 sample was directly cut by a single device process. For the $2016 \mathrm{~W}$ sample, a 0.38 -mm-wide cutter was used to cut over the phosphor layer. Then, the white silicon paste was molded onto the samples. The white silicon paste is composed of white color BN powder and the high-purity silicon paste. The detailed process can be seen in Fig. 1(a), that is, spraying the

(a)

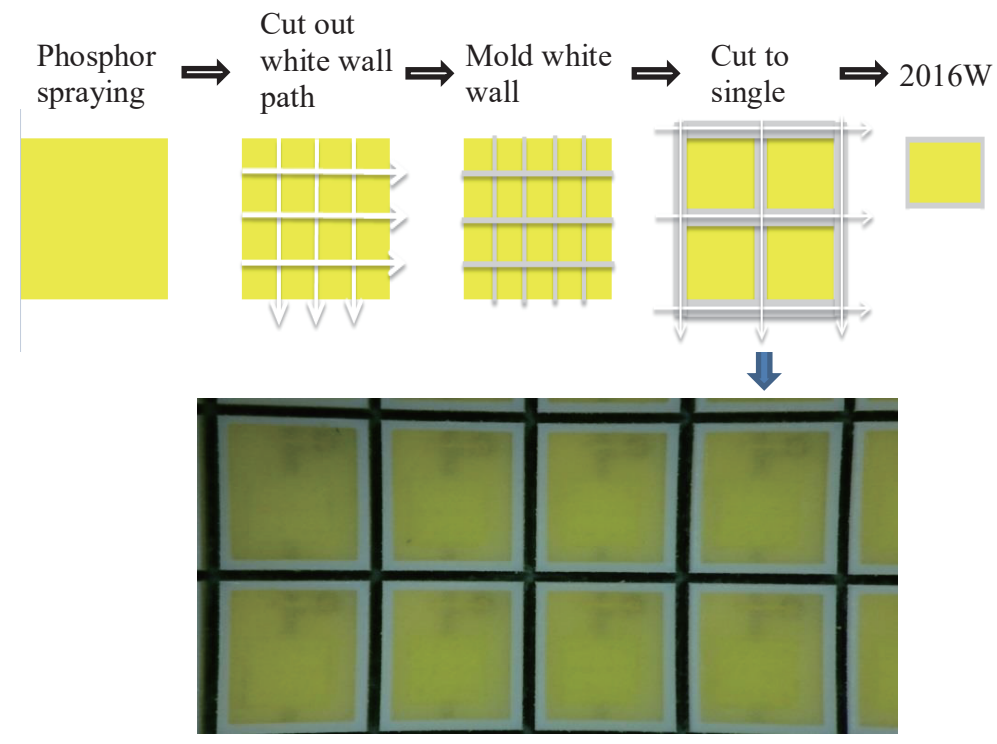

Fig. 1. (Color online) (a) Process flow of the phosphor molding for $2016 \mathrm{~W}$ and (b) finished sample after the white wall molding process. 
phosphor, cutting the phosphor paste, molding the white wall, and cutting the lead frame to a single device. The top view of the finished sample 2016W is shown in Fig. 1(b).

For the enhanced molding sample, 2016EW, two cutting processes were performed as shown in Fig. 2(a). A 0.5-mm-wide cutter was used to remove the phosphor layer on top of the Zener diode, and a 0.2-mm-wide cutter was used isolate the Zener diode and the LED die. Thus, the white wall can be built between the Zener room and the LED, where the Zener diode and the LED die have heights of 100 and $150 \mu \mathrm{m}$, respectively. We proposed that the light emitting from the LED die can be reflected by the white wall. The finished sample after using the three cutters is shown in Fig. 2(b). Figure 3 shows the top view of the three finished samples, namely
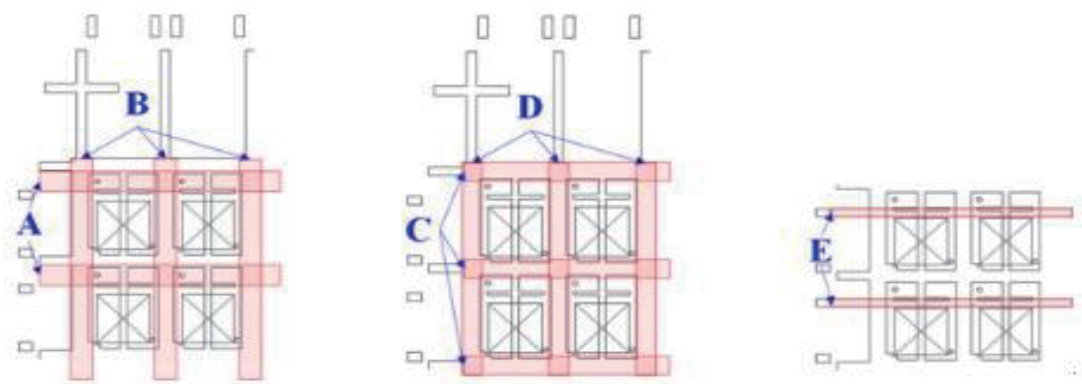

(a)

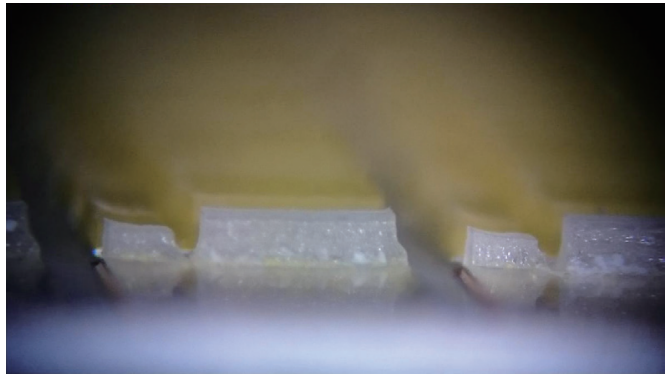

(b)

Fig. 2. (Color online) (a) Three steps of the phosphor removal processes for sample 2016EW (first cut, $0.5 \mathrm{~mm}$; second cut, $0.38 \mathrm{~mm}$; third cut, $0.2 \mathrm{~mm}$ ) and (b) before the white wall molding process.

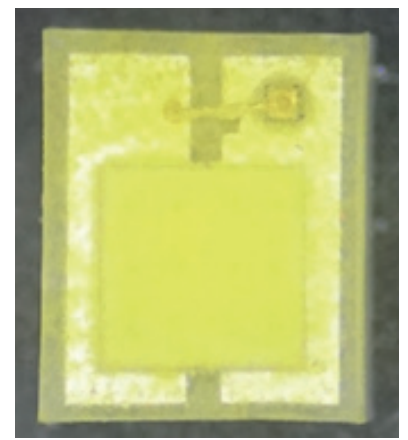

(a)

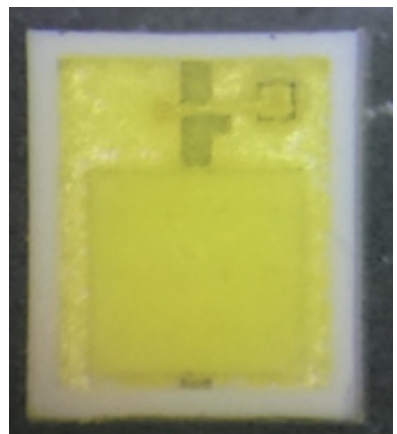

(b)

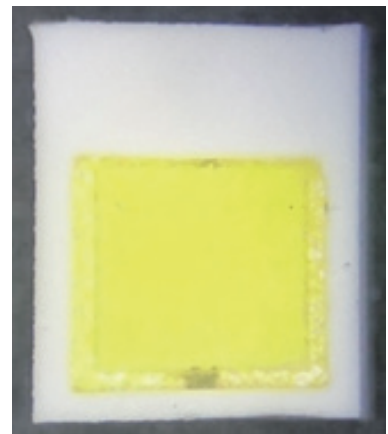

(c)

Fig. 3. (Color online) Top view of the three finished samples (a) 2016, (b) 2016W, and (c) 2016EW. 
2016, 2016W, and 2016EW, where the 2016W and 2016EW samples are with the same wall heights of about $0.5 \mathrm{~mm}$.

\section{Results and Discussion}

After finishing the above three types of package, i.e., samples 2016, 2016W, and 2016EW, the optical properties were examined using the integration sphere and the Keithley 2400 source meter. Figure 4 shows the luminous fluxes and luminous efficacies for samples 2016, 2016W, and 2016EW. Here, the total statistic number of the measured samples in our experiments was about $10 \mathrm{k}$. It was found that the average luminous fluxes were 272.09, 289.38, and $300.88 \mathrm{~lm}$ for samples 2016, 2016W, and 2016EW, respectively. When the LED was driven at a current of $1 \mathrm{~A}$, the lumen value for the enhanced white wall sample was increased by about $10.6 \%$ compared with that without the white wall. It also seems to have a larger error bar appearance in sample 2016W. It might be the blue light going through the Zener diode region during emission. In the contrast, the smaller error bar was achieved for sample 2016EW, which has an isolation white wall between the LED die and the Zener diode. Therefore, the samples with the extra isolation reflective walls not only enhanced the luminous performance significantly but also improved the reliability of the package process. In addition, it is worth pointing out that with the help of the white wall, the lateral loss of photons can be reduced significantly. Therefore, the amount of phosphor paste used to match the same level of chromaticity should be reduced. In our experiments, the usage of phosphors decreased to about $25 \%$ for the white wall case. Furthermore, the phenomenon of leakage blue light can be avoided for those LEDs with white wall processes. Figure 4 also shows the luminous efficacies of the above three samples. The luminous efficacies were $81.21,88.12$, and $92.1 \mathrm{~lm} / \mathrm{W}$ for samples 2016, 2016W, and 2016EW, respectively. Compared with that of sample 2016 without the white wall, the luminous efficacies were increased by about $8.5 \%$ and $13.4 \%$ for the white wall $2016 \mathrm{~W}$ and the enhanced white wall 2016EW samples, respectively.

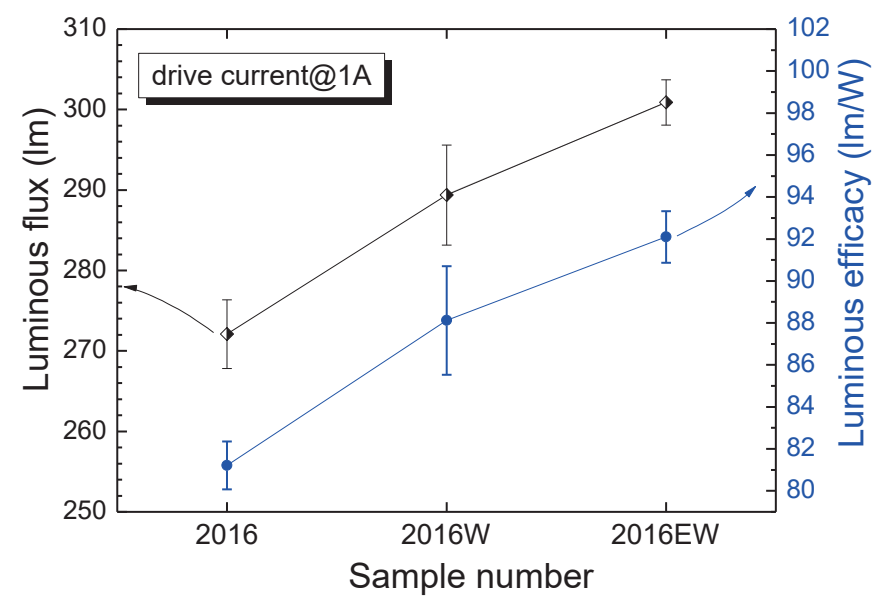

Fig. 4. (Color online) Luminous flux and efficacy for samples 2016, 2016W, and 2016EW. 


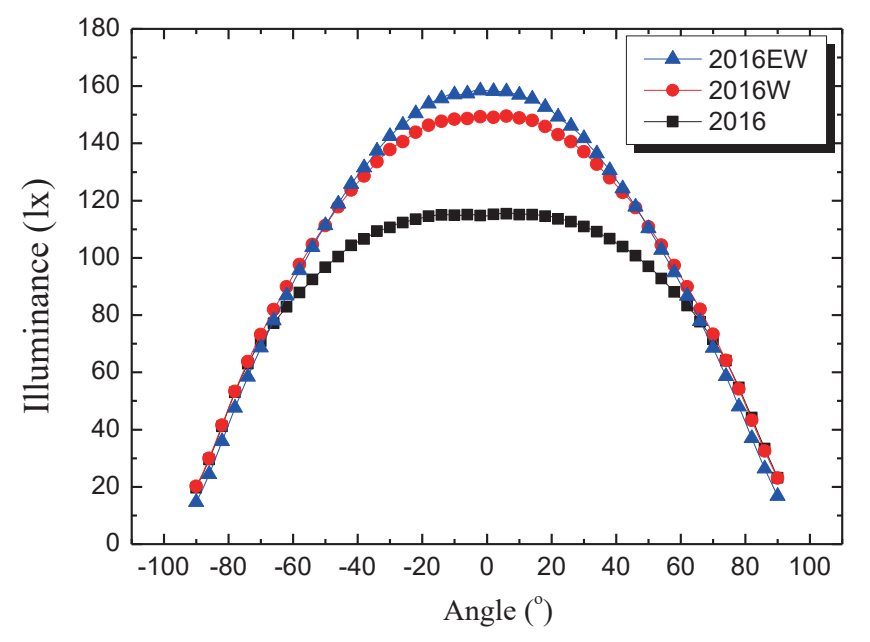

Fig. 5. (Color online) Illuminances of samples 2016, 2016W, and 2016EW.

For the application of flashlights in cell phones, the requirement of high illuminance and long distance at a short time is a very rigorous condition. Therefore, enhancement of the illuminance is an important task for developing a flashlight used in cell phone. Here, with the above mentioned three samples, the illuminances were measured in varying angles, as shown in Fig. 5. The illuminances in the normal direction (at zero degree) were 115.57, 149.47, and 158.43 lx for samples 2016, 2016W, and 2016EW, respectively. The illuminances were improved by about 29.3 and $37 \%$ for the $2016 \mathrm{~W}$ and the $2016 \mathrm{EW}$ samples, respectively. The reason is that the emitting photons were reflected back to the center region by the white wall. Therefore, the luminous flux per unit area was thus improved in the normal direction.

\section{Conclusions}

In summary, we proposed three types of packages of high-power LEDs for flashlight application. The light intensity was increased with the white wall process. It was found that the luminous fluxes were 272.09, 289.38, and $300.88 \mathrm{~lm}$ for samples 2016, 2016W, and 2016EW, respectively. In addition, the corresponding luminous efficacies for samples 2016, 2016W, and 2016EW were $81.21,88.12$, and $92.1 \mathrm{~lm} / \mathrm{W}$, respectively. Furthermore, the illuminance was also measured in varying angles. The illuminances at zero degree were 115.57, 149.47, and 158.43 lx for samples 2016, 2016W, and 2016EW, respectively. The reason is that the emitting photons were reflected back to the center region by the white wall. Therefore, the luminous flux per unit area was thus improved in the normal direction.

\section{Acknowledgments}

This work was partially supported by National Formosa University under contract number 106-AF-019. 


\section{References}

1 J. K. Sim, K. Ashok, Y. H. Ra, H. C. Im, B. J. Baek, and C. R. Lee: Curr. Appl. Phys. 12 (2012) 494.

2 J. K. Park, H. D. Shin, Y. S. Park, S. Y. Park, K. P. Hong, and B. M. Kim: 56th Electronic Components and Technology Conf. 2006 (IEEE, 2006) 1070-1075.

3 S. J. Chang, W. S. Chen, S. C. Shei, T. K. Ko, C. F. Shen, Y. P. Hsu, C. S. Chang, J. M. Tsai, W. C. Lai, and A. J. Lin: IEEE Trans. Adv. Packag. 30 (2007) 752.

4 S. J. Chang, C. S. Chang, Y. K. Su, C. T. Lee, W. S. Chen, C. F. Shen, Y. P. Hsu, S. C. Shei, and H. M. Lo: IEEE Trans. Adv. Packag. 28 (2005) 273.

5 L. Fan, Q. Liu, C. Jiang, H. Xu, J. Hu, D. Luo, Z. He, and Q. Huang: 2016 IEEE MTT-S Int. Wireless Symp. (IWS) (IEEE, 2016) 1-4.

6 X. Luo and S. Liu: IEEE Trans. Adv. Packag. 30 (2007) 475. 